\title{
Efficacy of a diet containing caseinate hydrolysate on signs of stress in dogs
}

\author{
Clara Palestrini $^{a}$, Michela Minero ${ }^{a}$, Simona Cannas ${ }^{a}$, Greta Berteselli ${ }^{a}$, \\ Elisabetta Scaglia ${ }^{a}$, Sara Barbieri ${ }^{a}$, Elena Cavallone ${ }^{b}$, Maria Puricellic, \\ Francesco Servida', Paola Dall'Ara ${ }^{c}$
}

\author{
${ }^{a}$ Dipartimento di Scienze Animali, Sezione di Zootecnica Veterinaria, Facoltà di Medicina Veterinaria, Università degli \\ Studi di Milano, Milano, Italy; \\ ${ }^{b}$ Laboratorio Centralizzato Radioisotopi, Facoltà di Medicina Veterinaria, Università degli Studi di Milano, Milano, Italy; \\ and \\ ${ }^{c}$ Dipartimento di Patologia Animale, Igiene e Sanità Pubblica Veterinaria, Sezione di Microbiologia e Immunologia, \\ Facoltà di Medicina Veterinaria, Università degli Studi di Milano, Milano, Italy.
}

\section{KEYWORDS:}

dogs;

stress;

anxiety;

caseinate hydrolysate;

heart rate;

cortisol

\begin{abstract}
The purpose of this randomized, double-blind, placebo-controlled trial was to evaluate the efficacy of a diet containing caseinate hydrolysate $(\mathrm{CH})$ on signs of stress in 2 groups of dogs (defined as Anxious and Nonanxious), using physiological (serum cortisol and lysozyme, N:L ratios and heart rate) and behavioral parameters.

From an initial group of 40 female Beagle dogs, ranging in age from 10 months to 4 years (mean = 1.47 years; $\mathrm{SD}=0.53$ ) belonging to a dog colony, 32 were selected for this study according to their level of anxiety. A group of 16 Anxious dogs and a group of 16 Nonanxious dogs were identified.

A baseline period, aimed to obtain reference values of investigated parameters, preceded the experimental phase. Both groups (Anxious and Nonanxious) were divided into a treatment group, which received the diet containing $\mathrm{CH}$, and a control group which received a placebo diet (PD). Anxious $\mathrm{CH}$ and $\mathrm{PD}$ groups were balanced for anxiety level. Each dog was evaluated 3 times a day at 4 weeks intervals (T1-T2-T3). Each evaluation lasted 2 days and involved a Reactivity Evaluation Form, a blood sampling, heart rate recording, and a 10-minute behavioral video recording. Results from Reactivity Evaluation Form scores showed that although at T1 Anxious dogs had significantly higher scores (Mann-Whitney test, $P<0.001$ ) compared with Nonanxious dogs, no difference was found between Anxious dogs fed with $\mathrm{CH}$ diet and Nonanxious fed with PD or $\mathrm{CH}$ diet at T3. Behavioral observations evidenced some signs of improvement in Anxious dog fed with $\mathrm{CH}$ diet. Cortisol level significantly decreased in Anxious dogs fed with $\mathrm{CH}$ diet (Friedman test, $P<0.05$ ). Individual differences in physiological measures of stress responses may have contributed to the large variability, making interpretation of these measures difficult. These results suggest that $\mathrm{CH}$ may be used as a functional ingredient alleviating stress in dogs.
\end{abstract}

(C) 2010 Elsevier Inc. All rights reserved.
Address for reprint requests and correspondence: Clara Palestrini, Dipartimento di Scienze Animali, Sezione di Zootecnica Veterinaria, Facoltà di Medicina Veterinaria, Università degli Studi di Milano, Via Celoria 10, 20133, Milano, Italy.

E-mail: clara.palestrini@unimi.it

\section{Introduction}

In the field of veterinary behavioral medicine, responses to stress and anxiety play an important part in the development of a large variety of behavioral "problems" in 
domestic cats and dogs (Casey, 2002). The term "anxiety" is used when an animal anticipates a negative outcome. Anxiety is an emotional response occurring before a stimulus or situation which the animal perceives as inevitable or dangerous (Neilson, 2002). Thus, when anxious, the animal exhibits a somatic and behavioral stress response to a situation or stimuli which might occur.

The stress response is an adaptive system which maximize an animal's ability to respond to an internal or peripheral challenge initiating physiological and behavioral changes (freeze-fight-flight), with the aim of preparing an animal to respond maximally to the particular situation (Casey, 2002).

Physiological changes include an increase in activity of the autonomous sympathetic system and reduction of the parasympathetic system with consequent vasodilatation in the vital organs, increased heart rate, and cardiac output accompanied by faster breathing, and decreased activity of the gastrointestinal and reproductive organs. In a stressful condition, the adrenal gland secretes epinephrine, norepinephrine, and steroid stress hormones (Carlson, 1994).

Behavioral responses of dogs to situations they perceive as stressful are avoidance, aggressiveness, panting, salivation, pacing, hyperactivity, hypervigilance, elimination, dilated pupils, lowered posture, hiding, digging, flattened ear, anorexia, attention-seeking, lip-licking, frequent swallowing, low tail position, vocalizing, and trembling (Beerda et al., 1998; Rooney et al., 2007).

The stress response system is best adapted for enabling animals to deal with acute stressors that they are able to escape from or deal with, by using some other behavioral or internal response. The stress response, in other words, works ideally to enable an animal to respond to an environmental stressor by displaying an appropriate behavior pattern that effectively removes the animal from the source of stress and the stress response rapidly declines (Casey, 2002). Stress responses to external stimuli become problematic when an individual animal is unable to control the situation or to escape from the stressor through an appropriate behavioral response (Weiss, 1972).

In these instances, in which the physiological stress response becomes prolonged or chronic, there are negative effects on both the emotional and physiological health of the individual (Casey, 2002).

The effect of prolonged stress reaction on the immune system represents a redistribution of immune cells out of the circulation into peripheral areas (Paik et al., 2000). This response certainly helps to explain the apparent increase in peripheral autoimmune reactions that occur in chronic stress in human beings and in laboratory animals (Thomason et al., 2002), as well as the decrease in resistance to blood-borne infectious agents (Tuchscherer and Manteuffel, 2000). Furthermore, a prolonged release of high levels of cortisol can have wide-ranging negative effects on the body. In fact, the physiological effects of prolonged stress can include increased blood pressure, diabetes, infertility, decreased attention, inhibition of inflammatory responses, and changes in immune function (Casey, 2002).

When the stressor is chronic, the animals will also exhibit inappropriate or excessive behavioral responses, with the aim of lowering the level of a prolonged stress response and, consequently, its harmful effect (Dantzer and Mormede, 1981).

Hence, chronic stress can have several deleterious or pathologic consequences for the animal, such as the development of alternative behaviors, termed "displacement activities," that direct the animal's energy into another activity and the development of repetitive, stereotypical, or compulsive behaviors (Cameron et al., 2001).

An inability to regulate the stress response has been proposed as a critical factor in the pathophysiology of various stress-related disorders such as anxiety disorders (Sullivan et al., 1999).

Stress can be measured using physiological and behavioral parameters. Either of these alone can be indicative of a less than desirable state of psychological health, but the use of both together is considered to be a more reliable measure of stress or welfare (Mason and Mendl, 1993).

Dog owners are more and more sensitive to these stress behaviors and may wish to treat them. The use of nonpharmaceutical, natural biological compounds is sometimes preferred to psychotropic drugs.

Cow's milk has long been considered a beverage, with natural "tranquilizing" properties. Research has shown that some of the peptides present in the milk may have a calming effect (Beata et al., 2007).

A milk caseinate hydrolysate $(\mathrm{CH})$ and a bioactive decapeptide $\left(\alpha_{\mathrm{s} 1}\right.$-casein-[f91-f100], $\alpha$-casozepine), a fragment of this hydrolysate that has been spatially modeled (Lecouvey et al., 1997), have already shown an anxiolytic-like profile in the conditioned defensive burying test and in the elevated plus maze in rats, 2 well-known models used to study anxiolytic agents in rodents (Miclo et al., 2001). Moreover, the effect of caseinate hydrolysate on hemodynamic responses was evaluated in healthy human volunteers facing successive mental and physical stress situations (Messaoudi et al., 2004) and in the management of anxious disorders such as social phobia in cats (Beata et al., 2007).

The purpose of this balanced, double-blind, placebocontrolled trial was to evaluate the efficacy of a diet containing $\mathrm{CH}$ on signs of stress in 2 groups of dogs (defined as Anxious and Nonanxious), using physiological and behavioral parameters.

\section{Materials and methods}

\section{Subjects}

Forty female Beagle dogs from a larger colony, ranging in age from 10 months to 4 years $($ mean $=1.47$ years; 
Table 1 Reactivity Evaluation Form (REF)

Phase 1

Investigator is standing outside the box

Dog is seeking for contact, stands permanently against the fence

Dog is successively standing against the fence and slightly retreating

Dog is permanently retreating but presents some attempts in seeking contacts

Dog is prostrated in the back of the box, standing as far as possible from the fence, no attempt in seeking contacts

Phase 2

Investigator comes inside the box and stays static without interacting with the dog

Dog is seeking for physical contact with the investigator

Dog ends up by having a physical contact with the investigator after a few attempts

Dog attempts to approach the investigator but does not dare to have a physical contact

Dog is prostrated in the back of the box, standing as far as possible from the fence, no attempt in seeking contacts

1

2

Items

\begin{tabular}{lll}
\hline Freezing & No $=0$ \\
Trembling & Yes $=1$ & No $=0$ \\
Yawning & Nos $=0$ \\
Lip-liking & Yes $=1$ & No $=0$ \\
Crouched & Yes $=1$ & No $=0$ \\
Tail between legs & Yes $=1$ & No $=0$ \\
Ears down & Yes $=1$ & No $=0$ \\
Defecation/urination & Yes $=1$ & No $=0$ \\
\hline
\end{tabular}

$\mathrm{SD}=0.53)$, were entered in this study. The dogs were kept in a shelter in a number of $2-3$ dogs per pen $(3 \times 4 \mathrm{~m})$. All the dogs were born in the colony and kept in the same environmental conditions.

The dogs were all considered healthy on the basis of complete physical examinations and clinical blood measures.

The experimental protocol was in compliance with the European Communities Council Directive of November 24, 1986 (86/609/EEC) for animal experiments.

\section{Data collection}

\section{Selection}

Each dog was evaluated in their own fenced pen by 2 experienced investigators already known by the animals (1 animal keeper and 1 veterinarian), using an Evaluation Scale of Anxiety and a Reactivity Evaluation Form (REF). The Evaluation Scale used was a continuous rating scale (McDowell, 2006), in which investigators rated each dog's anxiety level by placing a mark on a line with scale points under the line, from 1 (low level of anxiety)-6 (high level of anxiety). A grade was hence attributed to each dog.

The REF consisted of 2 parts: the first part considered the dogs' reactions to investigators while they were outside the pen and the second one while they were inside (Table 1). The REF was based on preliminary observations and then formulated considering the available literature on this topic (Dreschel and Granger, 2005; Waibelinger et al.,
2006; Frank, 2007; Overall et al., 2009; Sforzini et al., 2009).

During the first part, investigators were standing motionless at a distance of $50 \mathrm{~cm}$ in front of the pen for 2 minutes, avoiding any interactions with the dogs, and filled in the first part of the REF independently.

During the second part, both investigators entered the pen, closed the gate, and crouched with their back against the fence (avoiding any movement, direct gaze, and interaction with the dogs). For each part, based on the dogs' reactions and behaviors, evaluators were asked to score the dogs by selecting the most appropriate form items as shown in Table 1.

Measures included ordinal and dichotomous parameters. Results of individual reviews were compared and only little differences between the 2 operators were found. When there were little discrepancies between assessments made by the 2 operators, they were discussed and amended. After the scoring, each dog was characterized by a grade derived from the Evaluation Scale of Anxiety and by a score from the REF.

From the initial group of 40 dogs, a group of 16 Anxious dogs with the highest scores (obtained summing the Evaluation Scale of Anxiety and the REF) and a group of 16 Nonanxious dogs with the lowest scores were selected.

On the basis of the REF scores, dogs were sorted in decreasing order, from the most anxious dog to the least anxious, within each group (Anxious and Nonanxious). The 32 dogs where then allocated to 16 pens by pair so that each pair was made of dogs with similar scores. In this way, an Anxious dog with a high score of anxiety was associated with 
another Anxious dog with about the same level of anxiety. The same procedure was applied for Nonanxious dogs.

The decision to allocate dogs in pairs with similar scores was intended to avoid the behavior of one dog influencing the behavior of the other dog. Observational learning or social learning (learning what to do in a given situation from observing the behavior of another individual in that situation) may, in fact, be a significant factor affecting the behavior of dogs (Mills, 2002). Dogs had 3 days to acclimate to their new cagemate and were fed with their usual food every evening at the same hour "ad libitum" with a feedbox. Pens were cleaned every day.

\section{Baseline}

The baseline period lasted 6 days and aimed to obtain reference values of serum cortisol, serum lysozyme, white blood cell counts to assess N:L (neutrophils:lymphocytes) ratios and mean heart rate for all 32 dogs. Each blood sample was taken in jugulars veins (right or left) with a "venoject" system (single use needle-vacutainer tube) and done outside the pens between 2and 4 P.M Dogs were restrained minimally while blood was collected in $3 \mathrm{~mL}$ EDTA tubes and $4 \mathrm{~mL}$ in a serum tube. White blood cells counts were done on the same day as collection. All blood samples for cortisol and lysozyme assays were centrifuged within 1 hour after collection and the serum was frozen at $-20^{\circ} \mathrm{C}$ until being analyzed.

Cortisol was measured by a competitive radioimmunoassay validated for canine species (Coat-A-Count TKCO, Diagnostic Products Corporation, DPC, Los Angeles, U.S.A.).

Lysozyme concentration was determined using a micromethod assay set up at the Department of Veterinary Animal Pathology and Public Health (Section of Microbiology and Immunology, University of Milan, Italy) starting from the classic lysoplate method of Osserman.

A suspension of Micrococcus lysodeikticus cell walls (Sigma) at a final concentration of $0.6 \mathrm{mg} / \mathrm{mL}$ in $1 \%$ low electroendosmosis and sulfate content agarose prepared in $0.066 \mathrm{M}$ phosphate buffer, $\mathrm{pH}$ 6.6, was poured in aliquots of $50 \mathrm{~mL} \times 15 \mathrm{~cm}$ diameter Petri dishes.

Just before use, 44 wells, $3-\mathrm{mm}$ in diameter, were punched into the gel with a cork borer and evacuated with a Pasteur pipet connected to a water aspirator.

Samples and standard solutions (20-10-5-2.5-1.25-0.625 $\mu \mathrm{g} / \mathrm{mL}$ ) from chicken egg white lysozyme (Sigma), prepared in the same buffer used for the preparation of the gel medium, were placed in the wells in duplicate $(10 \mu \mathrm{L} /$ well $)$.

After incubation of the plates at $24^{\circ} \mathrm{C}$ for 18 hours, the diameter of the cleared zone of lysis was measured with a special ruler.

Exponential standard curves from the size of the cleared zones of the lysozyme standard solutions, from which the lysozyme concentration in the unknowns was estimated, were used.

Baseline heart rate measurement was telemetrically performed inside their own pen. Heart rate was measured using a recording heart rate device (Polar® RS 400), at 5second intervals. The Polar ${ }^{\circledR}$ was fixed around the chest of the animal with an elastic band. During the baseline period all the dogs were fed their usual diet.

\section{Experimental phase}

The feeding intervention phase lasted 60 days immediately after an initial 3-day evaluation phase (T1). The feeding phase consisted of 4-week periods with 2-day evaluation phases (T2, T3) after each of the 4 weeks.

Each group (Anxious and Nonanxious) was divided into a control group and a treatment group. The treatment group received the diet containing caseinate hydrolysate and control group received a placebo diet (PD). Within the Anxious and Nonanxious groups, each pen was randomly assigned to treatment or control diet. Final groups were subsequently controlled for anxiety level and they were balanced.

For the duration of the study, dogs were fed every evening at the same hour "ad libitum" with a feedbox. Food was always available. Daily intake was recorded and bodyweight was measured weekly. Food composition was protein $25 \%$, crude fat $10 \%$, starch $41.6 \%$, dietary fiber $9.9 \%$, crude fiber $4.2 \%$, moisture $8 \%$, and metabolizable energy $3792 \mathrm{kcal} / \mathrm{kg}$ - NRC 74-for placebo diet (PD). The same composition, with the adjunction of $\mathrm{CH}$ containing alpha casozepine s1 after extrusion and coated on the core kibble to prevent it from destruction through extrusion process, was used for the $\mathrm{CH}$ diet.

To check the level of $\mathrm{CH}$ in the diet an external tracer (yttrium oxide) was used. In particular, $42 \mathrm{ppm}$ of yttrium oxide was added i to reach $35 \mathrm{ppm}$ of yttrium in the diet. Yttrium oxide was homogeneously blended into $\mathrm{CH}$. The blend of $\mathrm{CH}$ and yttrium oxide was coated on the core kibbles through a batch coating system. Ten samples of $100 \mathrm{~g}$ kibble were taken from the batch and used to build a $100 \mathrm{~g}$ sample representative of the batch. Yttrium was analyzed at $34 \mathrm{ppm}$ on the average sample which represents 3\% quantity loss from the initial dosage which was considered acceptable. Both diets were packaged in indistinguishable bags with a code corresponding to the number of the pen.

Each evaluation (T1, T2, and T3) lasted 2 days. During the first day, the REF was filled in using the same procedure described in paragraph 2.2.2 and a blood sample was taken. During the second day, heart rate measurements and a video recording lasting 10 minutes were performed.

During these procedures all the experimenters were blinded to the treatment given.

The video room, situated in the same building as the dogs, was equipped with a bowl with food $(\mathrm{CH}$ diet or PD based on the group), a bowl of water, and a toy. A video camera was positioned in a pen next to the video room.

The Polar $^{\circledR}$ (portable heart rate monitor; Polar Electro Oy, Kempele, Finland), set at a 5-second interval, was wrapped around the dog's chest and synchronized to the video recording. The dog was left free to explore the room and filmed for 10 minutes. 


\section{Data analysis}

All the data were transcribed to Excel files and statistically analyzed with SPSS 16.0 (SPSS, 2007). The level of significance was set to $P<0.05$.

\section{Evaluation scale and REF}

Subjective scores of the anxiety level of dogs (from very low to very high level of anxiety) were obtained from the Evaluation scale of anxiety. Answers to the REF were coded and individual dogs' scores were calculated by summing up phase scores and related items.

Correlations (Spearman's rho) between the scores of the Evaluation Scale of Anxiety and the REF were calculated to evaluate the agreement between them.

A discriminant analysis on REF scores was used to test how well individual dogs were classified as Anxious or Nonanxious.

To compare Anxious and Nonanxious dogs fed with different diets a descriptive statistic, Friedman and MannWhitney tests were used.

\section{Behavior (video)}

The behavior of each dog was video-recorded in the video room and the 10-minute videotape sessions were analyzed by a trained observer, blinded to treatment and groups. Sixteen categories covering all recorded behaviors were established (Table 2). A focal animal continuous recording method (Martin and Bateson, 1993) was used to describe the dog's activity.

Behaviors recorded as states and expressed in terms of duration (exploration, locomotion, passive, oriented to the environment, scratching, oral behavior, vocalization, grooming, panting, playing, and others) were reported as the average of the total observation time, and events (yawning, lip-licking, elimination, drinking, and eating) were reported in terms of absolute frequency of occurrence.

Descriptive measures (mean, median, SD) were calculated to describe behaviors shown during the 3 repeated video-recordings.

A Mann-Whitney nonparametric test was performed to compare Anxious and Nonanxious dogs at T1. Friedman and Wilcoxon tests were used to analyze how behavior varied over the 3 repetitions (T1-T2-T3).

\section{Cortisol}

The comparison of the cortisol level in Anxious and Nonanxious dogs between baseline and $\mathrm{T} 1$ was performed using a Wilcoxon test.

Differences among $\mathrm{T} 1, \mathrm{~T} 2$, and $\mathrm{T} 3$ in the different groups were evaluated by Friedman and Wilcoxon nonparametric tests.

Table 2 Behavioral categories and their definition

\begin{tabular}{|c|c|}
\hline Behavioral category & Definition \\
\hline \multicolumn{2}{|l|}{ Duration } \\
\hline Exploration (EX) & $\begin{array}{l}\text { Motor activity directed toward physical aspects of the environment, including } \\
\text { sniffing, and gentle oral examination such as licking }\end{array}$ \\
\hline Locomotion (LO) & Walking or running around without exploring the environment \\
\hline Passive behavior (PA) & $\begin{array}{l}\text { Lying down with the head on ground without any obvious orientation toward } \\
\text { the physical or social environment }\end{array}$ \\
\hline Oriented to the environment $(\mathrm{OE})$ & $\begin{array}{l}\text { Sitting, standing or lying down (the head does not rest on the ground) with obvious } \\
\text { orientation toward the physical or social environment, including sniffing, close visual } \\
\text { inspection, distant visual inspection (vigilance or scanning) }\end{array}$ \\
\hline Scratching (SC) & $\begin{array}{l}\text { All active behaviors resulting in physical contact with the cage, including scratching } \\
\text { the cage with the paws, jumping on the cage, handling with the forelimbs }\end{array}$ \\
\hline Oral behavior (OB) & $\begin{array}{l}\text { Any vigorous behavior directed toward the environment/cage using the mouth } \\
\text { (including chewing, biting, shaking, pulling with the mouth) }\end{array}$ \\
\hline Vocalization (V0) & Barking, whining and howling \\
\hline Play (PL) & $\begin{array}{l}\text { Any vigorous or galloping gaited behavior directed toward a toy; including chewing, } \\
\text { biting, shaking from side to side }\end{array}$ \\
\hline Panting (PT) & Painting \\
\hline Grooming (GR) & $\begin{array}{l}\text { The action of cleaning of the body surface by licking, nibbling, picking, rubbing, } \\
\text { scratching, etc. directed towards the animal's body (self-grooming) }\end{array}$ \\
\hline Other behaviors & Any activity not included in the behavioral categories \\
\hline \multicolumn{2}{|l|}{ Frequency } \\
\hline Yawning (YA) & Yawning \\
\hline Lip-licking (LL) & Part of tongue is shown and moved along the upper lip \\
\hline Elimination (EL) & Defecation or urination in sitting or standing position \\
\hline \multicolumn{2}{|l|}{ Drinking (DR) } \\
\hline Eating (ET) & \\
\hline
\end{tabular}




\section{Other physiological parameters}

Data distribution of lysozyme, N:L ratios, and heart rate was analyzed and found normal. To describe how these parameters changed over time for Anxious and Nonanxious dogs, a General Linear Model (GLM) analysis of variance for repeated measures including $\mathrm{T} 1, \mathrm{~T} 2$, and $\mathrm{T} 3$, was performed using the diet as a fixed factor and repetition as within subjects factor.

\section{Results}

REF values and investigated physiological parameters did not change significantly between baseline and $\mathrm{T} 1$, in both Anxious and Nonanxious dogs. Hence, T1 was considered as baseline.

\section{Evaluation scale and REF}

REF showed a positive significant correlation with the Evaluation Scale (Spearman's rho $=0.837 ; P<0.001$ ).

The discriminant analysis proved that, overall, the model was correct (Canonical correlation $=0.66$; Wilks's $\lambda P<$ 0.001 ): based on the posterior probability for belonging to the predicted group, 3 anxious dogs were not properly classified and therefore eliminated in the subsequent statistical analyses. Final group sizes were 14 Anxious dogs and 16 Nonanxious.

At T1 Anxious dogs had significantly higher REF scores (Mann-Whitney test, $P<0.001$ ) compared to Nonanxious dogs, and scores of Anxious dogs fed with placebo diet (PD) did not differ from scores of Anxious dogs fed with $\mathrm{CH}$ diet (Figure 1).

REF values were affected by treatment. At T3 REF values of Anxious dogs fed with $\mathrm{CH}$ diet decreased significantly over time (Friedman test, $P<0.01$ ).

\section{Behavior (video)}

At T1 Anxious dogs did not show any significant difference in duration and frequency of the recorded behaviors from Nonanxious (Table 3). Exploration, oriented to the environment, vocalization, and scratching were the most frequently observed behaviors.

Scratching behavior decreased over time (Friedman test, $P<0.05)$ in Nonanxious dogs fed with PD, whereas liplicking increased (Friedman test, $P<0.05$ ) in Anxious dogs fed with PD. Anxious dogs, fed with $\mathrm{CH}$ diet, had significantly decreased exploratory behavior and were progressively more oriented to environment, significantly differing at $\mathrm{T} 1$ and $\mathrm{T} 3$ (Friedman test, $P<0.05$ ) (Figures 2 and 3 ).

Behaviors like locomotion and passivity were exhibited by both Anxious and Nonanxious dogs only in T3. Although Anxious dogs fed with $\mathrm{CH}$ diet spent more time in passive behaviors compared to Anxious dogs fed with PD, no significant differences were found.

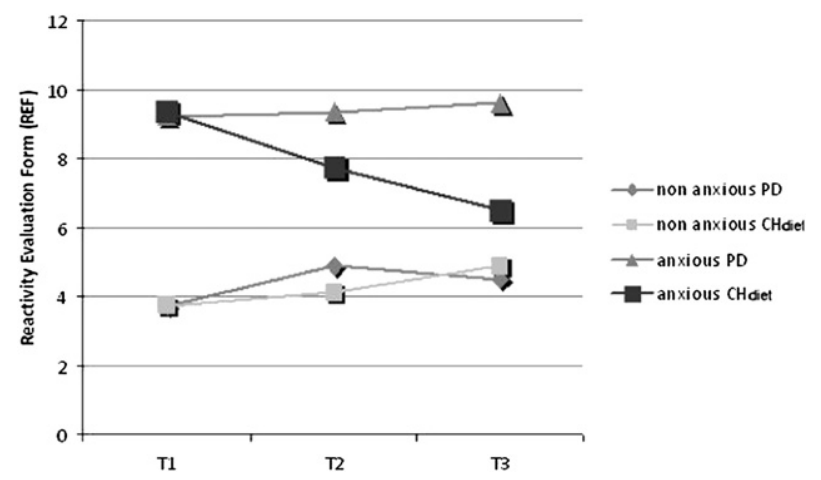

Figure 1 Reactivity Evaluation Form (REF) scores of the different groups at T1, T2, and T3.

\section{Cortisol}

Plasma cortisol basal levels were in the normal physiological range in both Anxious and Nonanxious dogs over entire duration of study (from 13.79 to $165.54 \mathrm{nmol} / \mathrm{L}$ ) (Feldman and Nelson, 2004). At T1 cortisol in Anxious and Nonanxious dogs did not differ significantly.

The evaluation of differences in cortisol plasma level among T1, T2, and T3 showed a significant decrease in Anxious dogs fed with $\mathrm{CH}$ diet (Friedman test, $P<0.05$ ) and a tendency to decrease in Nonanxious dogs fed with $\mathrm{CH}$ diet (Friedman test, $P<0.10$ ).

In particular, Anxious dogs fed with $\mathrm{CH}$ diet showed a significant decrease in cortisol between $\mathrm{T} 1$ and $\mathrm{T} 2$ (Wilcoxon test, $P<0.05$ ) (Figure 4).

\section{Other physiological parameters}

In both Anxious and Nonanxious dogs lysozyme increased significantly over time (T1, T2, T3) (GLM, $P<0.001$ ), but it was not affected by the diet.

In Anxious dogs neutrophils level decreased and lymphocytes level increased significantly (GLM, $P<0.05$ ) over time. On the contrary, in Nonanxious dogs N:L ratio did not vary over time. In both groups of dogs changes were not affected by the diet. No differences were found on the remaining physiological variables examined.

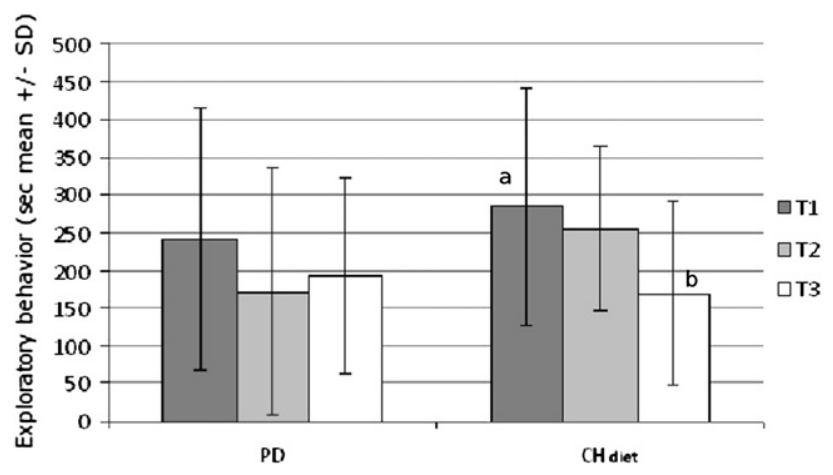

Figure 2 Exploratory behavior in anxious dogs at T1, T2, and T3 (mean \pm SD). Bars with different superscripts significantly differ. 
Table 3 Duration (sec) or frequency of recorded behaviors at T1

\begin{tabular}{|c|c|c|c|c|}
\hline \multirow[b]{2}{*}{ Behavioral category } & \multicolumn{2}{|l|}{ Nonanxious dogs } & \multicolumn{2}{|l|}{ Anxious dogs } \\
\hline & Duration (mean $\pm \mathrm{SD}$ ) & Frequency (mean \pm SD) & Duration (mean $\pm S D$ ) & Frequency (mean $\pm \mathrm{SD}$ ) \\
\hline EX & $262 \pm 140$ & - & $263 \pm 161$ & - \\
\hline $\mathrm{OE}$ & $200 \pm 170$ & - & $245 \pm 166$ & - \\
\hline $\mathrm{OB}$ & $31 \pm 61$ & - & $9 \pm 9$ & - \\
\hline SC & $34 \pm 28$ & - & $24 \pm 41$ & - \\
\hline V0 & $72 \pm 88$ & - & $46 \pm 83$ & - \\
\hline $\mathrm{GR}$ & - & - & $1 \pm 3$ & - \\
\hline LO & - & - & - & - \\
\hline PA & - & - & - & - \\
\hline$P L$ & - & - & $1 \pm 4$ & - \\
\hline PT & $1 \pm 3$ & - & - & - \\
\hline $\mathrm{LL}$ & - & $2 \pm 2$ & - & $2 \pm 4$ \\
\hline YA & - & - & - & $0 \pm 1$ \\
\hline ET & - & $0 \pm 1$ & - & - \\
\hline $\mathrm{DR}$ & - & - & - & - \\
\hline EL & - & - & - & $0 \pm 1$ \\
\hline
\end{tabular}

\section{Discussion and Conclusions}

The aim of the present study was to evaluate the efficacy of a diet containing $\mathrm{CH}$ on signs of stress in 2 groups of dogs (Anxious and Nonanxious) on the basis of behavioral and physiological parameters.

Several of the parameters used in this study have been reported to characterize the different components of a stress reaction: questionnaires (Kim et al., 2007), endocrine assay (Ilardo et al., 2001) and physiological measurements (Boiten et al., 1994; Larson et al., 2001).

Results from questionnaire-based studies show that $\alpha_{\mathrm{s} 1}$-casein hydrolysate may be used as an effective functional ingredient alleviating stress-related symptoms after 30 days of administration in women (Kim et al., 2007) and after 56 days in cats (Beata et al., 2007). Our results showed that a $\mathrm{CH}$ diet administered for a period of 65 days reduced some stress-related indicators in dogs.

The REF scores of Anxious dogs fed with $\mathrm{CH}$ diet decreased significantly over time. Specifically, the REF scores of Anxious dogs fed with $\mathrm{CH}$ diet were similar to those of Nonanxious dogs at T3, whereas Anxious dogs fed with PD continued to show scores that differed significantly from Nonanxious dogs.

Behavioral observations also suggested improvement in Anxious dogs fed with $\mathrm{CH}$ diet. Although at T1, Anxious and Nonanxious dogs behaved similarly, some behaviors changed significantly over time. Anxious dogs fed with $\mathrm{CH}$ diet spent significantly less time exploring and more time being oriented to the environment at T3. In contrast, it is possible to interpret decreasing exploration as a sign of anxiety (Crawley and Goodwin, 1980; Handley and Mithani, 1984), whereas an increase in motor activity (excessive activity, pacing, increased deambulation) is reported by several authors (Boissy, 1995; Lang et al., 2000; Casey, 2002) as a behavior related to stress and anxiety.
Normally, exploration is evoked by novel stimuli and consists of behavioral acts and postures that permit the collection of information about new objects and unfamiliar parts of the environment (Crusio, 2001). Over the course of the experimental phase, dogs were exposed to the same environment that gradually became known and familiar.

Adaptation to new objects and the environment can therefore be expressed with decreased exploration as opposed to decreased orientation to the environment and therefore interpreted as sign of reduced stress in the current study.

Furthermore, the frequency of lip-licking significantly increased in Anxious dogs fed with PD compared to all other groups, indicating a condition of persistent stress. Lip-licking has been reported as a stress-related behavior in dogs (Casey 2002; Frank et al., 2006) and has been associated with fear or anxiety (Beerda et al., 1998) or as a possible displacement behavior indicative of conflict (Voith et al., 1987).

Beerda et al. (1998), showed that dogs confronted with stressful stimuli in a social context could react by exhibiting oral behaviors such as sticking its tongue out, licking its snout, swallowing, and yawning (Beerda et al., 1998; Godbout et al., 2007).

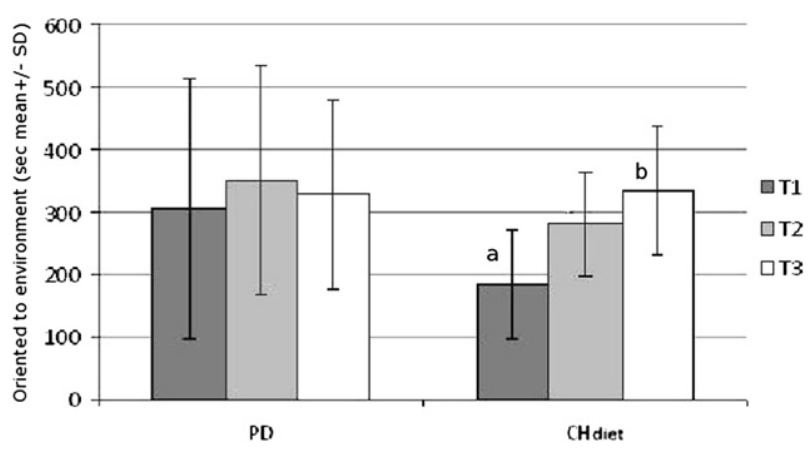

Figure 3 Oriented to the environment behavior in anxious dogs at $\mathrm{T} 1, \mathrm{~T} 2$, and $\mathrm{T} 3$ (mean $\pm \mathrm{SD}$ ). Bars with different superscripts significantly differ. 


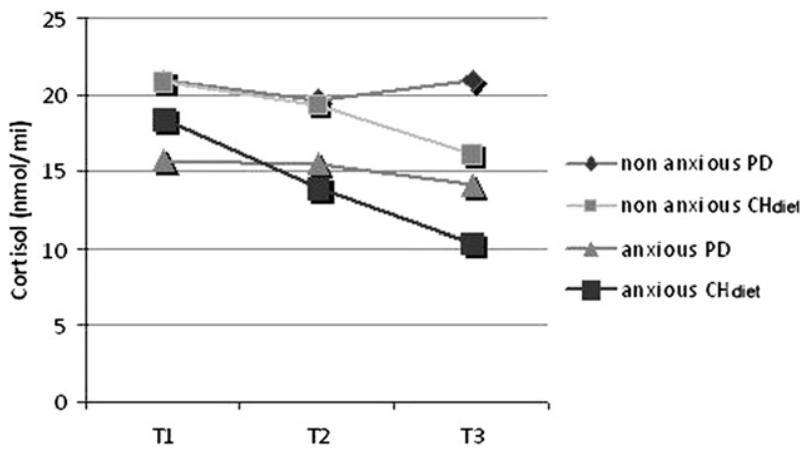

Figure 4 Plasma cortisol level in the different groups at T1, T2, and T3.

Scratching behavior also has been reported as a sign of distress, anxiety, or fear (Horwitz, 2002; Neilson, 2002; Overall, 2002). In our study, Nonanxious dogs fed with PD showed significantly decreased scratching behavior probably indicating a form of adaptation to a new environment.

Physiological measures of the stress response are advantageous in that they provide quantitative measures. However, not only is there large individual variation in physiological response to stressors, but also there are normal variations at different times of the day that can make comparison and interpretation difficult (Rushen, 1991).

Cortisol is normally released in a pulsatile manner (Ladewig, 1987); the plasma levels vary with diurnal rhythm (Walker et al., 1992). To minimize those factors in our study, each blood sample was taken at the same time of the day.

Although cortisol can be also measured in urine or feces, plasma concentration best reflects the stress response at the time of the measurement even though it can be influenced by the stress related to blood sampling (Casey, 2002).

Restraint and venipuncture may cause sufficient stress to increase baseline cortisol level when sampling is not performed promptly following restraint or if restraint is excessive (Hennessy et al., 1998; Frank et al., 2006). For this reason, all our animals had been trained for blood sampling and acquisition of other measures before the study started to ensure that the results were not biased by the procedure itself. Garnier et al. (1990) found that bitches had a greater cortisol response to a novel environment than male dogs. In this study, cortisol level significantly decreased in our female Anxious dogs fed with $\mathrm{CH}$ diet at T2 in spite of the new environment, supporting a possible improvement in Anxious dogs' stress conditions.

Results from other physiological parameters measured in this study are not easy to interpret. In both groups of dogs lysozyme increased significantly over time, but it was not affected by diet, demonstrating that all dogs, to some extent, adapted to the new condition, that is, when stress stimuli stop, lysozyme concentrations return to normal values (Yang and Glaser, 2000; Berteselli et al., 2005).

Our study showed a significant decrease in N:L ratio in Anxious dogs, while these parameters did not change in Nonanxious subjects.
Specific changes in the number of peripheral neutrophils, lymphocytes, and eosinophils may indicate acute stress in dogs. Whether changes in peripheral leucocytes sustain during chronic stress and prove to be valid indicators of poor physiological welfare, remains open to further investigation. Data on stress-induced changes in the canine immune system are scarce and limited to peripheral leucocyte counts.

It appears that acute stress induces an overall peripheral leucocytosis consisting of neutrophilia, lymphopenia, and eosinopenia. In our study, dogs did not face an acute stress, but a chronic anxiety problem: it is possible that under this condition the hematological system does not react with a "stress leukogram," but with less clear variations. Individual differences (Lawler et al., 1975; Koepke and Obrist, 1983) may further increase variability in stress responses and make interpretation even more difficult (Beerda et al., 1997). No difference was found in mean heart rate in Anxious and Nonanxious dogs over time.

Even though not all parameters showed statistical changes over time, there was a general tendency for a decrease in physiological responses associated with stress, as well as a decrease in the frequency or duration of some behaviors compatible with anxiety in Anxious subjects fed with $\mathrm{CH}$ diet compared with dogs fed with PD or Nonanxious dogs.

In conclusion, this study provided evidence that $\mathrm{CH}$ may be used as a useful functional ingredient for alleviating stress in dogs. Further studies are needed to investigate whether the conclusion drawn from this population can be generalized to dogs housed in different conditions or affected by different behavioral disorders and if the observed changes are maintained on a long-term basis.

\section{References}

Beata, C., Beaumont-Graff, E., Coll, V., Cordel, J., Marion, M., Massal, N., Marlois, N., Tauzin, J., 2007. Effect of alpha-casozepine (Zylkene) on anxiety in cats. J. Vet. Behav.: Clin. Appl. Res. 2, 40-46.

Beerda, B., Schilder, M.B.H., van Hoff, J.A.R., de Vries, H.W., Mol, J.A., 1998. Behavioural, saliva cortisol and heart rate responses to different types of stimuli in dogs. Appl. Anim. Behav. Sci. 58, 365-381.

Beerda, B., Schilder, M.B.H., van Hooff, J.A.R.A.M., de Vries, H.W., 1997. Manifestations of chronic and acute stress in dogs. Appl. Anim. Behav. Sci. 52, 307-319.

Berteselli, G., Servida, F., Dall'Ara, P., Verga, M., Piola, E., Puricelli, M., Palestrini, C., 2005. Evaluation of immunological and behavioural parameters in dogs with anxiety related disorders. In: Mills, D., Levine, E., Landsberg, G., Horwitz, D., Duxbury, M., Mertens, P., Meyer, K., Huntley, L.R., Reich, M., Willard, J. (Eds.), Current Issues in veterinary Behavioural Medicine Proceedings of the 5th International Veterinary Behavioural Meeting. Purdue University Press, Minneapolis, MN.

Boissy, A., 1995. Fear and fearfulness in animals. Q. Rev. Biol. 70, 165-191. Boiten, F.A., Frijda, N.H., Wientjes, C.J.E., 1994. Emotions and respiratory patterns: review and critical analysis. Int. J. Psychophysiol. 17, 103-128.

Cameron, M.E., Casey, R.A., Bradshaw, J.W., Waran, N., Gunn-Mooore, D., 2001. A study of the environment and behavioural factors involved in the triggering of idiopathic cystitis in the domestic cat. BSAVA Congress 2001 Scientific Proceeding, Birmingham, United Kingdom, p. 505.

Carlson, N.R., 1994. Physiology and Behavior. Allyn and Bacon, Massachusetts, MA, pp. 359-361. 
Casey, R., 2002. Fear and stress. In: Horwitz, D., Mills, D., Heath, S. (Eds.), BSAVA Manual of Canine and Feline Behavioural Medicine. BSAVA, Gloucester, United Kingdom, pp. 144-153.

Crawley, J., Goodwin, F.K., 1980. Preliminary report of simple animal behavior model for the anxiolytic effects of benzodiazepines. Pharmacol. Biochem. Behav. 13, 167-170.

Crusio, W.E., 2001. Genetic dissection of mouse exploratory behavior. Behav. Brain. Res. 125, 127-132.

Dantzer, R., Mormede, P., 1981. Pituitary adrenal consequences of adjunctive behaviours in pigs. Horm. Behav. 15, 386-395.

Dreschel, N.A., Granger, D.A., 2005. Physiological and behavioral reactivity to stress in thunderstorm-phobic dogs and their caregivers. Appl. Anim. Behav. Sci. 95, 153-168.

Feldman, E.C., Nelson, R.W., 2004. Canine hyperadrenocorticism. In: Canine and Feline Endocrinology and Reproduction, 3rd Ed. Saunders, Philadelphia, PA, pp. 252-357.

Frank, D., 2007. Drugs and behavior: what, when and why? Proceedings of the WSAVA Congress, Sydney, Australia.

Frank, D., Gauthier, A., Bergeron, R., 2006. Placebo-controlled double-blind clomipramine trial for the treatment of anxiety of fear in beagles during ground transport. Can. Vet. 47, 1102-1108.

Garnier, F., Benoit, E., Virat, M., Ochoa, R., Delatour, P., 1990. Adrenal cortisol response in clinically normal dogs and after adaptation to a housing environment. Lab. Anim. 24, 40-43.

Godbout, M., Beauchamp, G., Palestrini, C., Frank, D., 2007. Puppy behavior at the veterinary clinic: a pilot study. J. Vet. Behav.: Clin. Appl. Res. 2, 126-135.

Handley, S.L., Mithani, S., 1984. Effects of alpha-adrenoreceptor agonists and antagonists in a maze-exploration model of "fear"-motivated behavior. Naunyn. Schmiedebergs. Arch. Pharmacol. 327, 1-5.

Hennessy, M., Davis, H., Wiliams, M., Mellott, C., Douglas, C., 1998. Plasma cortisol levels of dogs at a county animal shelter. Physiol. Behav. 62, 485-490.

Horwitz, D.F., 2002. Separation-related problems in dogs. In: Horwitz, D., Mills, D., Heath, S. (Eds.), BSAVA Manual of Canine and Feline Behavioural Medicine. BSAVA, Gloucester, United Kingdom, pp. 154-163.

Ilardo, C., Toniolo, A.M., Aimone-Gastin, I., Abdelmouttaleb, I., Gueant, J.L., Desor, D., 2001. Effects of psycho-physiological stress (competitive rafting) on saliva interleukin-1 beta. Stress Health 17, 9-15.

Kim, J.H., Desor, D., Kim, Y.T., Yoon, W.J., Kim, K.S., Jun, J.S., Pyun, K.H., Shim, I., 2007. Efficacy of alpha s1- casein hydrolysate on stress-related symptoms in women. Eur. J. Clin. Nutr. 61, 536-541.

Koepke, J.P., Obrist, P.A., 1983. Angiotensin II in the renal excretory function during behavioural stress in conscious stress. Am. J. Physiol. 245, R259-R264.

Ladewig, J., 1987. Endocrine aspects of stress: evaluation of stress reactions in farm animals. In: Wiepkema, P.R., van Adrichem, P.W.M. (Eds.), The Biology of Stress in Farm Animals: An Integrated Approach. Martinus Nijhoff, Dordrecht, the Netherlands, pp. 13-25.

Lang, P.J., Davis, M., Ohman, A., 2000. Fear and anxiety: animal models and human cognitive psychophysiology. J. Affect. Disord. 61, 137-159.

Larson, M.R., Ader, R., Moynihan, J.A., 2001. Heart rate, neuroendocrine, and immunological reactivity in response to an acute laboratory stressor. Psychosom. Med. 63, 493-501.

Lawler, J.E., Obrist, P.A., Lawler, K.A., 1975. Cardiovalscular function during pre-advoiance, avoiance and post-avoiance in dogs. Psychophysiology $12,4-11$.

Lecouvey, M., Frochot, C., Miclo, L., Orlewski, P., Marraud, M., Gaillard, J.L., Thong Cung, M., Vanderesse, R., 1997. Conformational studies of a benzodiazepine-like peptide in SDS micelles by circular dichroism, H NMR and molecular dynamics simulation. Let. Pept. Sci. 4, 359-364.

Martin, P., Bateson, P., 1993. Measuring Behaviour: An Introductory Guide, 2nd Ed. Cambridge University Press, Cambridge, United Kingdom, pp. 84-85.
Mason, G., Mendl, M., 1993. Why there is no simple way of measuring animal welfare? Anim. Welf. 2, 301-319.

McDowell, I., 2006. Measuring Health: A Guide to Rating Scales and Questionnaires. Oxford University Press, New York, NY.

Messaoudi, M., Lefranc-Millot, C., Desor, D., Demagny, B., Bourdon, L., 2004. Effects of a tryptic hydrolysate from bovine milk aplha s1-casein on hemodynamic responses in healthy human volunteers facing successive mental and physical stress situations. Eur. J. Nutr. 44, 128-132.

Miclo, L., Perrin, E., Driou, A., Papadopoulos, V., Boujrad, N., Vanderesse, R., Boudier, J.F., Desor, D., Linden, G., Gaillard, J.L., 2001. Characterization of alpha-casozepine, a tryptic peptide from bovine alpha s1-casein with benzodiazepine-like activity. FASEB J $15,1780-1782$.

Mills, D.S., 2002. Learning, training and behavior modification techinques. In: Horwitz, D., Mills, D., Heath, S. (Eds.), BSAVA Manual of Canine and Feline Behavioural Medicine. BSAVA, Gloucester, United Kingdom, pp. 37-48.

Neilson, J.C., 2002. Fear of places or things. In: Horwitz, D., Mills, D., Heath, S. (Eds.), BSAVA Manual of Canine and Feline Behavioural Medicine. BSAVA, Gloucester, United Kingdom, pp. 173-180.

Overall, K.L., 2002. Noise phobias in dogs. In: Horwitz, D., Mills, D., Heath, S. (Eds.), BSAVA Manual of Canine and Feline Behavioural Medicine. BSAVA, Gloucester, United Kingdom, pp. 164-172.

Overall, K.L., Juarbe-Diaz, S., Dyer, D.J., Chang, M.L., Yokoyama, J.S., Dunham, A.E., 2009. Assessing reactivity in 3 breed of working dogs: phenotypic determination and associated genotypes. J. Vet. Behav.: Clin. Appl. Res. 4, 249.

Paik, I.H., Lee, C., Kim, J.J., Lee, S.J., 2000. Psychological stress may induce increased humoral and decreased cellular immunity. Behav. Med. 26, 139-141.

Rooney, N.J., Gaines, S.A., Bradshaw, J.W., 2007. Behavioural and glucocorticoid responses of dogs (Canis familiaris) to kenneling: investigating mitigation of stress by prior habituation. Physiol. Behav. 92, 847-854.

Rushen, J., 1991. Problems associated with the interpretation of physiological data in the assessment of animal welfare. Appl. Anim. Behav. Sci. 28, 381-386.

Sforzini, E., Michelazzi, M., Spada, E., Ricci, C., Carenzi, C., Milani, S., Luzi, F., Verga, M., 2009. Evaluation of young and adult dogs' reactivity. J. Vet. Behav.: Clin. Appl. Res. 4, 3-10.

SPSS., 2007. SPSS 16.0 Command Syntax Reference. SPSS Inc., Chicago, IL.

Sullivan, G.M., Coplan, J.D., Kent, J.M., Gorman, J.M., 1999. The noradrenergic system in pathological anxiety: a focus on panic with relevance to generalized anxiety and phobias. Biol. Psychiat. 46, 1205-1218.

Thomason, J.J., Brantley, P.J., Jones, G.N., Dyer, H.R., Morris, J.L., 2002. The relations between stress and disease activity in rheumatoid arthritis. J. Vet. Med. 15, 215.

Tuchscherer, M., Manteuffel, G., 2000. The effect of stress on the immune system. Another reason for pursuing animal welfare (review). Arch. Tierz. 43, 547-560.

Voith, V.L., Borchelt, P.L., 1987. Elimination behaviour and related problems in dogs. Veterinaria 1, 47-56.

Waiblinger, S., Boivin, X., Pedersen, V., Tosi, M.V., Jancza, K.A.M., Visser, E.K., Jones, R.B., 2006. Assessing the human-animal relationship in farmed species: a critical review. Appl. Anim. Behav. Sci. 101, $185-242$.

Walker, C.D., Lightman, S.L., Steel, M.K., Dallaman, M.F., 1992. Suckling is a persistent stimulus to the adreno-cortical system of the rat. Endocrinology 130, 115-125.

Weiss, J.M., 1972. Psychological factors in stress and disease. Sci. Am. 226, 104-113.

Yang, E.V., Glaser, R., 2000. Stress-induced immunomodulation: impact on immune defenses against infectious disease. Biomed. Pharmacother. $54,245-250$. 